\title{
Wearable, Redundant Fabric-Based Sensor Arrays for Reconstruction of Body Segment Posture
}

\author{
Federico Lorussi, Walter Rocchia, Enzo Pasquale Scilingo, Alessandro Tognetti, and Danilo De Rossi
}

\begin{abstract}
Posture and gesture analysis, together with the monitoring of body kinematics, is a field of increasing interest in bioengineering and several connected disciplines. In this paper, some typical features of distributed sensing systems are described, as well as a methodology to read signals from such systems. Theory, simulation, results, and some specific applications are shown. Strain gauges have been used as sensors and have been deposited directly onto textile fibers, demonstrating one way to realize a wearable sensor system.
\end{abstract}

Index Terms-Electroactive polymers, posture reconstruction, redundancy in sensing systems, resistive network reading algorithms, smart textiles.

\section{INTRODUCTION}

$\mathbf{T}$ HE FABRICATION of electronic systems onto substrates which are not only flexible, but also conformable to the human body, represents a breakthrough in many areas of application, such as virtual reality, teleoperation, telepresence, ergonomics, and rehabilitation engineering [1]. The possibility of realizing sensing textiles by coating traditional fabrics with smart materials (piezoresistive, piezoelectric, and piezocapacitive polymers) is quite recent and has opened up a means of implementing a new type of man-machine interface technology [2]. Peculiar features that require the application of new processing approaches have then emerged. Having a set of sensors distributed on a garment poses a certain number of new problems, amongst which is the need for minimizing the wiring required to extract the signals from every single sensor. Second, given the variability between individuals, the sensors on a garment cannot, in general, always be positioned exactly at the same location; therefore, the repeatability of measurements is not guaranteed on the same subject, and it becomes entirely different going from one subject to another. An even more demanding requirement resides in the need for a high immunity to motion artifacts and for provisions to deal with the sensors crosstalk. A basic point made here is based on the observation that a redundant number of sensors distributed on a surface

Manuscript received November 8, 2002; revised January 11, 2004. This work was supported in part by DARPA and in part by ONR-IFO under NICOP Grant N00014-01-0280 PR N 01PR04487-00. The associate editor coordinating the review of this paper and approving it for publication was Prof. Ralph-Etienne Cummings.

F. Lorussi, E. P. Scilingo, and A. Tognetti are with the Interdepartmental Research Center E. Piaggio, University of Pisa, Pisa 56126, Italy (e-mail: f.lorussi@ing.unipi.it; e.scilingo@ing.unipi.it; a.tognetti@ing.unipi.it).

W. Rocchia is with NEST-INFM Scuola Normale Superiore, Pisa 56126, Italy (e-mail: w.rocchia@sns.it).

D. De Rossi is with the Interdepartmental Research Center E. Piaggo, University of Pisa, Pisa 56126, Italy, and also with the CNR Institute of Clinical Physiology, Pisa 56126, Italy (e-mail: derossi@ing.unipi.it).

Digital Object Identifier 10.1109/JSEN.2004.837498

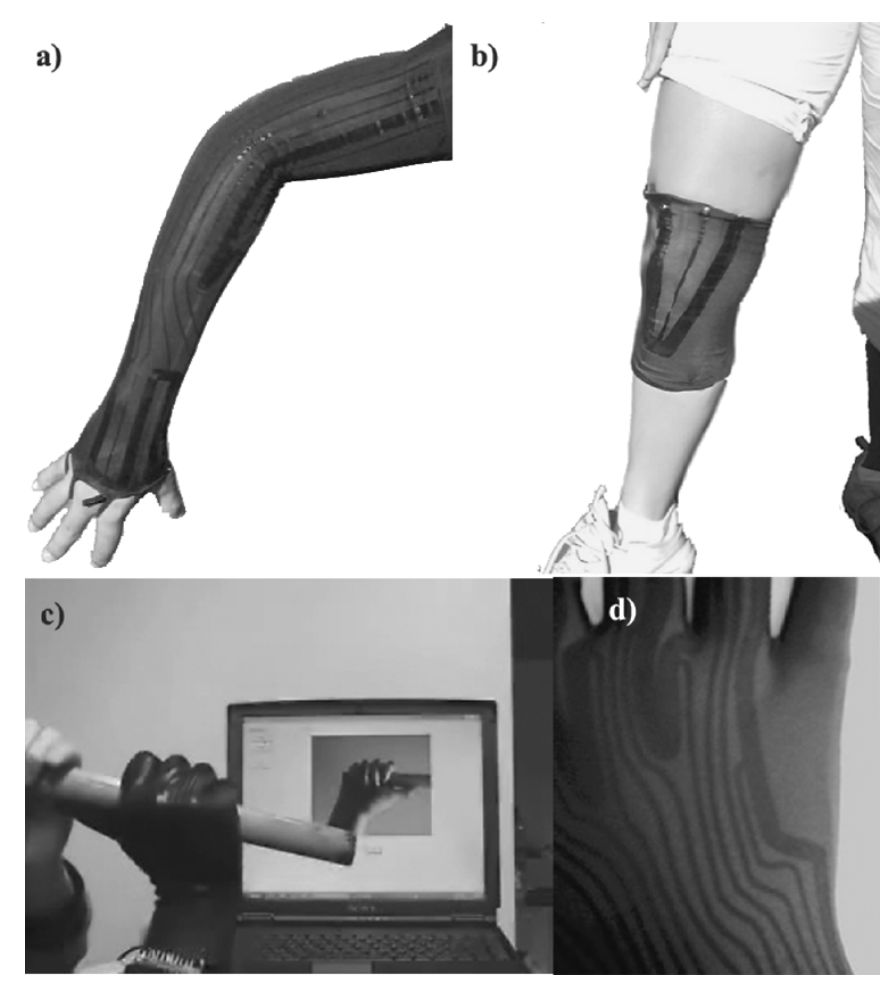

Fig. 1. Prototypes of sensing wearable devices. (a) Sensing sleeve. (b) Sensing knee pad. (c) Sensing glove during the recognition of a power grasp. (d) Detail of sensing and conducting tracks on a sensing fabric.

can provide enough positional information to infer the essential features concerning the posture of a subject, also loosening the constraint of precise sensor location. This approach borrows from the biological paradigm. For instance, as far as mechanoreception is concerned, high-quality biological sensors are not used everywhere, particularly if they are sufficiently numerous [5]. On the other hand, reading singularly a very numerous set of sensors can be time consuming and it requires a noticeable amount of wiring, unless suitable strategies are adopted. In this paper, we illustrate the techniques which enable the realization and utilization of wearable sensing garments capable of recording proprioceptive maps with no discomfort for the subject and negligible motion artifacts caused by sensor-body mechanical mismatch. In Sections II-V, some issues concerning the realization of sensing textiles, the topology, and the reading strategy are faced and a possible way of approaching them is presented. In Sections VI-VIII, it is described why it can be convenient to directly map the space of resistances into the space of postures rather than passing through the single-sensor deformation space. 


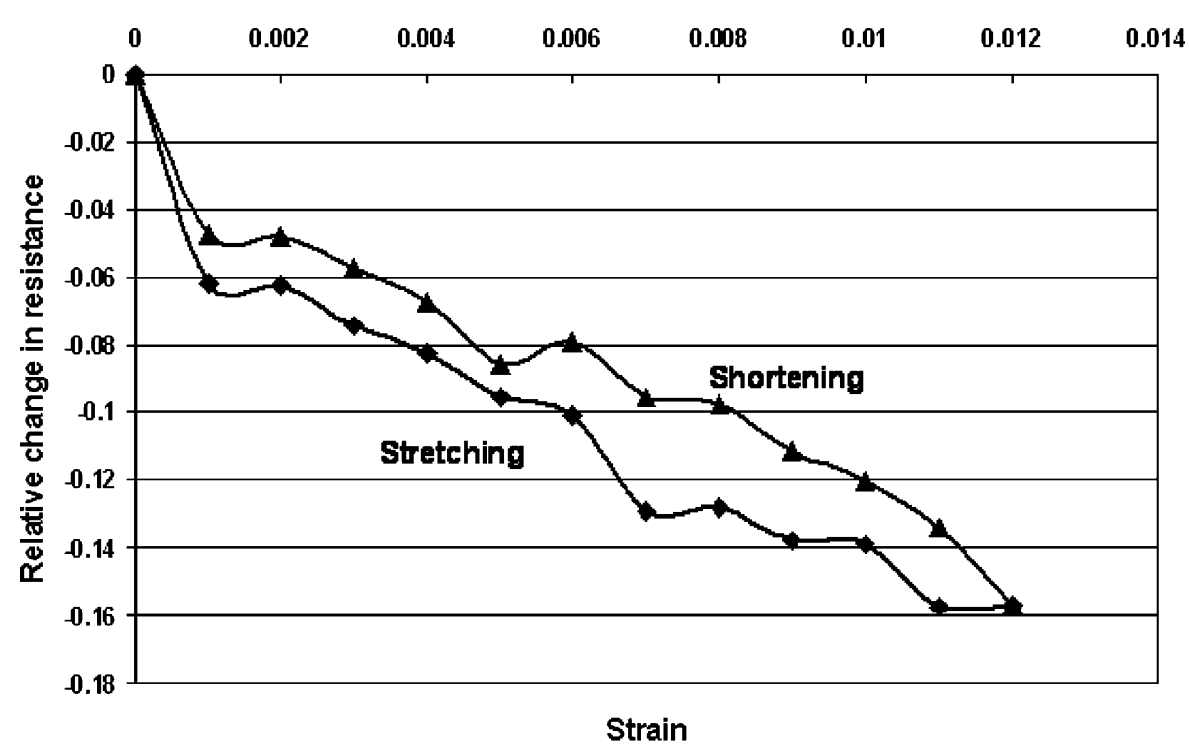

Fig. 2. Quasi-static response in terms of percent change in electrical resistance versus uniaxial strain (both stretching and shortening) for a PPy-based sensor.

\section{SENSING FABRic REALIZATION}

Elastic fabrics covered with an epitaxial layer of conducting polymer or with carbon-filled rubber show piezoresistive properties, and they can be used to realize sensorized garments, such as gloves, leotards, seat covers, and related artifacts capable of reconstructing and monitoring body shape, posture, and gesture [6]. Sensors have been realized starting from conventional fabrics coated with a thin layer of polypyrrole (PPy, a П-electron conjugated conducting polymer) or by a mixture of rubber and carbon. PPy is a conducting polymer that combines good environmental stability with mechanical and thermal transduction capabilities. PPy-coated lycra fabrics were prepared using the method reported in reference [7]. Sensors based on carbonfilled rubber (CFR) were realized either by directly printing the carbon/rubber mixture onto fabrics or by weaving CFR coated fibers. Threads of this type have been obtained as an experimental product by Smartex s.r.l. (Prato, Italy); fabrics have been coated in our laboratories. The first three prototypes we realized, shown in Fig. 1, were meant to be able to detect arm, knee, and hand posture. The more advanced state of analysis is currently concerning the "sensing sleeve" and, therefore, in this paper, we will show mainly results arising from arm posture detection.

\section{A. Sensors Characterization}

The sensors were characterized in terms of quasi-static and dynamic electromechanical transduction properties. Thermal and aging properties of the sensing fabrics have also been assessed. The characterization of PPy coated fabrics has shown a gauge factor (GF $=\Delta R / \epsilon R_{0}$, where $\delta R$ is the variation of the sensor resistance, $R_{0}$ its rest value and $\epsilon$ is the applied strain) of about -13 (negative and similar to nickel) and a temperature coefficient of resistance (TCR) of about $0.018 \mathrm{C}^{-1}$. In Fig. 2, a quasi-static response in terms of percent change in electrical resistance versus uniaxial strain, both in stretching and shortening configuration, for a PPy-based sensor is reported. It can be noted that a hysteresis loop is present, i.e., the fabric sensor response when it is stretched lies on a different path against shortening solicitation. The sensor response is also affected by noise artifacts. The noise disturbance has been estimated as an uncertainty band on the signal coming from the fabric sensors. This implies, obviously, that the intrinsic noise of the sensor is not decoupled from interferences due to electronic devices used for the acquisition. In the light of these considerations, the estimated error signal is about $3 \%$.

Despite the fact that a high GF value is a positive factor for strain gauge realization, two serious problems affect PPy-coated fabric sensors. The first problem resides in the strong variation with time of the sensor resistance. The second problem is the long sensor response time; in fact, after a sudden application of a mechanical stimulus, the resistance reaches a steady state in several minutes; this makes these fabrics unusable in most applications. Nevertheless, these limitations have been partially overcome by an "ad hoc" coding procedure. Analyzing the resistance response in the range of $1 \mathrm{~s}$ after the application of a stepwise deformation, it is possible to derive the applied strain in an aging invariant way. We experimentally observed that the area between the characteristic response to a strain step function and the line representing its asymptotic behavior remains constant throughout the aging process. A specific proof has been reported in [8], leading to the conclusion that the mentioned area codifies for the strain regardless of sensor aging process.

Analogously to PPy sensors, in Fig. 3, the quasi-static response in terms of percent change in electrical resistance versus strain (both stretching and shortening) for a CFR-based sensor is reported. Similarly, a hysteresis cycle is present. Even in this case, we evaluated the noise, as overall disturbance coming from the system fabric sensor plus electronics, and we observed an error signal of $2.5 \%$.

The characterization of CFR-coated fabrics has shown a GF of about 2.5 and a TCR of roughly $0.08 \mathrm{C}^{-1}$. These values are quite similar to those of metals and are suitable for the use of such sensors in wearable applications.

CFR fabrics age very slowly and they behave like low-pass filters with a bandwidth from dc to $8 \mathrm{~Hz}$. A thorough dynamic sensor characterization has also been performed, but it goes beyond the scope of this paper and can be found in [9]. 


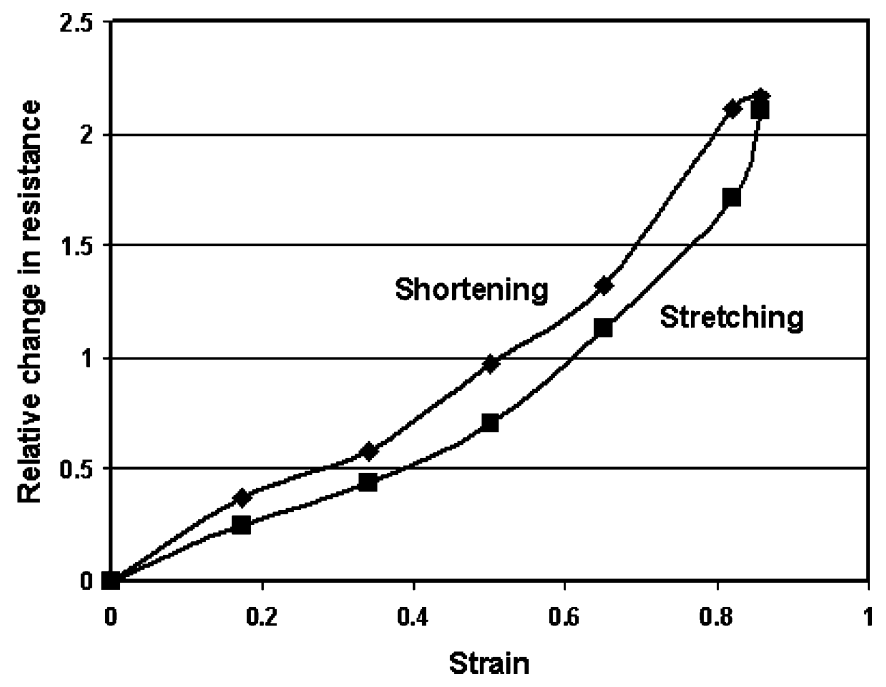

Fig. 3. Quasi-static response in terms of percent change in electrical resistance versus strain (both stretching and shortening) for a CFR-based sensor.

\section{SENSOR ARRAYS}

As said in Section II, in the last few years, textile technology progressed toward a design philosophy of integrating embedded computation and sensing into usual garments. Nowadays, it is possible, indeed, to realize sensorized gloves, leotards, and seat covers. Since the aim of these devices is to monitor body shape, posture, and gesture, a helpful approach is to rely on a redundant set of sensors suitably distributed. Several manufacturing textile processes could be implemented for use in sensorized garments. By using commercial embroidery processes, it is possible to stitch sensing surfaces and conductive threads defining a matrix pattern [10]. Alternative methods consist of using screenprinting technologies, in which strain sensors, e.g., CFR-based sensors, are applied according to predetermined masks. Sensors of the fabric matrix are interconnected by means of conductive threads. Cross points are avoided by means of insulating layers. In the case of a fabric matrix, a crucial problem is to read the sensors in a robust, reliable, and convenient way. Having a large number of sensors provides the necessary information, even if every single reading is not extremely accurate. In fact, this leads to a great versatility in device realization. Single-sensor reading increases the complexity of the electronic acquisition system dramatically. In order to address this problem, we have studied several topologies of sensor interconnections to reduce the number of sampling channels and tracks. Several types of interconnection have been studied. They use a bipolar model for each sensor. The first two strategies consist of interconnecting arrays of sensors in series (Fig. 4) or parallel (Fig. 5) networks. The two configurations are topologically dual.

Pairs of sensors (whose distance is negligible with respect to the geometric dimension of the net) on the fabrics are connected rows by columns. The inevitable crossing of wires makes it necessary to electrically insulate them to realize series and parallel connections.

By reading a variation of a column and of a row, it is possible to identify a precise point in the net. To do this, we have to assume that sensor variation occurs at any one time. In these

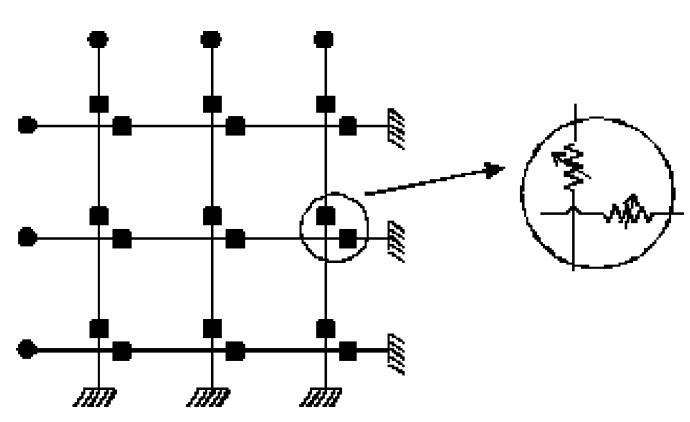

Fig. 4. Series network. The small distance between the two sensors ensures that their deformation is the same and differs from the other couples.

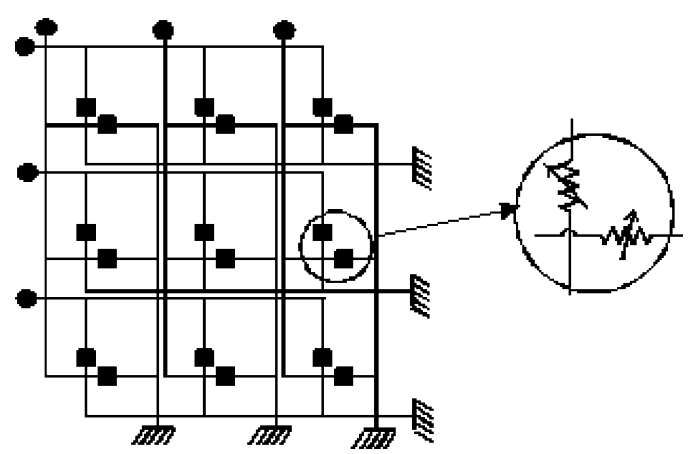

Fig. 5. Parallel network. A deformation of a pair of sensors implies a resistance variation of the entire row and column identifying the couple.

two configurations, we consider a square array of sensors and an $N$-channel acquisition system can read a network of $(N / 2)^{2}$ pairs of sensors. With respect to a single-sensor reading, these two topologies are advantageous (reduced the number of channels) if $N \geq 6$, even if they lead, as will be discussed later on, to a loss in the accuracy of signal reconstruction. Assuming we read sensor resistance variations by voltage $(V)$ variations with an assigned constant current, we define sensor sensitivity as $\delta V / \delta \epsilon$ and accuracy in signal reconstruction as $\Delta V / V_{\text {bias }}$ (where $V_{\text {bias }}$ is the voltage read for unloaded sensors and $\epsilon$ is the applied strain). Assuming each channel includes a certain number $(k)$ of identical sensors and assuming that only a single pair of sensors undergoes deformation, the expression of the voltage $(V)$ read from the channel with an imposed constant current $(i)$ is

$$
V=\mathrm{ik} R\left(1+\frac{\Delta R}{k R}\right)+\mathrm{ke}=\mathrm{ik} R\left(1+\frac{\mathrm{GF}}{k} \epsilon\right)+\mathrm{ke}
$$

for the series network. $R$ is the sensor resistance, $\Delta R$ is its variation, GF is the single-sensor gauge factor, and $\epsilon$ is the rms value of the resultant of intrinsic noise and interference on each sensor. For the parallel network, we have

$$
\begin{aligned}
V & =i \frac{R}{k}\left(1+\frac{\Delta R}{k R+(k-1) \Delta R}\right)+e \\
& =i \frac{R}{k}\left(1+\frac{\mathrm{GF} \epsilon}{k+(k-1) \mathrm{GF} \epsilon}\right)+e .
\end{aligned}
$$

Table I shows the expressions of sensitivity and accuracy for the single-sensor reading strategy and the two bipolar ones.

Even though the series network exhibits the better sensitivity and accuracy, both topologies have several limitations due to the 
TABLE I

SENSITIVITy AND ACCURACY FOR DifFERENT SENSOR READING STRATEGIES

\begin{tabular}{l|l|l}
\hline Configuration & Sensitivity & Accuracy \\
\hline Single sensor & $i R G F$ & $G F \epsilon$ \\
\hline Series & $i R G F$ & $G F \epsilon / K$ \\
\hline Parallel & $\frac{i R G F}{[k+(k-1) G F \epsilon]^{2}}$ & $\frac{G F \epsilon}{[k+(k-1) G F \epsilon]}$ \\
\hline
\end{tabular}

strong condition that sensors variations occur at any one time, in order to univocally determine the point in the network in which sensor response changes. An alternative strategy, usually used in an acquisition system based on matrix configuration, is to introduce some logical circuitry in order to sequentially scan each channel [11]. By using suitable digital switches, indeed, it would be possible to read the signals at boundaries of the network, but directly addressing the single sensors. However, this configuration involves a careful scanning timing, as well as a supplementary hardware to place onto garments and an increasing of the number of connections. These shortcomings make this approach unsuited for our purposes. Summarizing, none of the strategies described in this section are appropriate to detect complex patterns of superficial deformation, with the exception of a single-sensor reading scheme.

\section{Sensing Surface Strain Fields}

Hereafter, the problem of inferring the space varying resistance value on a conductive surface is solved making only measurements at the surface borders. A very similar problem arises in the field of electrical impedance tomography, where the resistive properties of a body are investigated by applying electrodes on its surface. In the works of Cheney et al., the issue is first described in its continuum formulation, based on Maxwell and constitutive laws; then, the discretized version, more physically sound due to the nature of the measuring electrodes, is formally derived [3], [4]. Here, we deal with a two-dimensional (2-D) problem and consider both situations, the discretization process of an actual 2-D conductivity distribution, such as a conductive membrane with electrodes on its border and the intrinsically discrete configuration where many sensors are positioned on an insulating 2-D scaffold and are connected through a wiring that respects the topology of the mesh described below. We will follow this latter case, where the issue of nonlinearity induced by discretized contacts as described in [4] is overcome. The final result is a lumped parameter bipolar mesh that, for static analysis, can be thought of as being purely resistive. If the associated graph is planar, then the mesh can be recast and converted in a square mesh, as the one shown in Fig. 6.

The practical realization of this network is simplified from the fact that connections are realized between adjacent sensors and no insulated cable is needed. This configuration is the less burdensome in terms of wiring. If there are $N$ nodes on a side, $4(N-1)$ channels are needed to record the signals from the border. It is actually possible to infer the strain field from border readings, and a proof for this is provided in the Appendix. In the next section, we present an algorithm that derives the conductance values from border readings through linear combinations of the read potentials. One or more currents are injected externally to the network and the potential values at the border

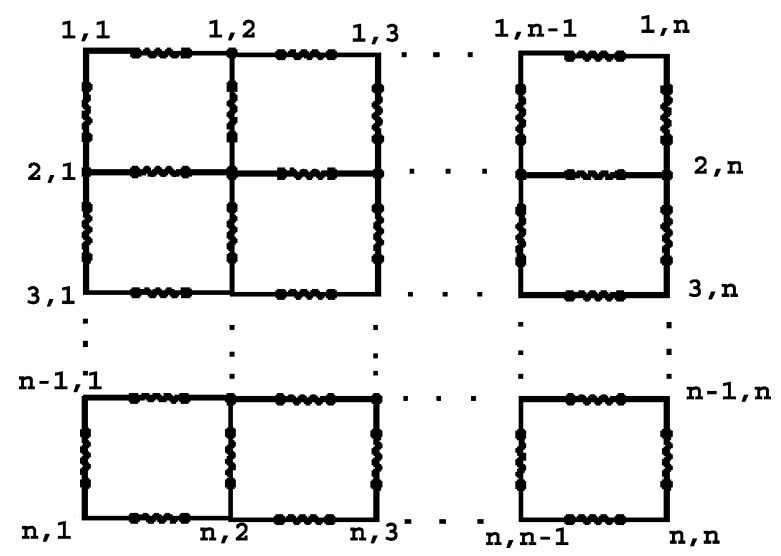

Fig. 6. Schematic of a discretized model for a distributed conductivity function.

nodes are read, one of them being kept as a reference. This algorithm results from an attempt to improve the accuracy in the reconstruction of the conductance value from possibly inaccurate border measurements. Beyond the different geometry, the present one being based on a square rather than circular geometry, this algorithm is different from the NOSER algorithm described in [3] in the sense that it has been devised in a way to admit a symbolic solution. The foreseeable advantages are diverse, the main one being that the solution obtained this way is expected to be particularly robust as far as accuracy is concerned; this is of particular importance, considering the degradation of accuracy occurring when one considers resistance values far from the mesh border. Along with it, convergence issues typical of numerical nonlinear programming algorithms are avoided. In order to design the algorithm, the simulation has been performed with a specific tool for symbolic calculation (MAPLE $V^{\mathrm{TM}}$ ) and both injected current and measured potential values have been converted from floating point to rational numbers. To solve the problem of identifying the conductances of each resistor, the so-called incidence matrix approach has been adopted, which is frequently used in software for simulating electrical circuits. Let $N$ be the number of nodes on each side of the square mesh and $N^{2}$ the overall number of nodes. The total number of internal branches $B=2 N(N-1)$ is augmented by $Q=2(N-1)(4 N-5)$ external inputs, required to inject external currents. As a first assignment, the latter correspond to all the possible combinations of external branches; this is instrumental in studying the best inputs and the effects of redundancy in input assignment on the accuracy in conductance determination. The topology of the mesh is represented by the $N^{2} \times(B+Q)$ incidence matrix A whose elements are 1 if the corresponding side is entering the current node, and -1 if it is exiting that node, and 0 otherwise. Operations with this matrix are always symbolic and do not introduce numerical inaccuracy on the involved data, even when floating-point data are used. Now, the fundamental equations for the circuit are

$$
\begin{aligned}
A \underline{I} & =\underline{0} \\
\underline{V} & =A^{T} \underline{\Phi} \\
\underline{I} & =G \underline{V}-\underline{I_{0}}
\end{aligned}
$$


representing Kirchoff's and constitutive laws, where $\underline{I}$ is the $(B+Q)$-dimensional vector containing all the currents of the circuits, $\underline{I}_{0}$ contains only the injected currents, $\underline{V}$ is the $(B+$ $Q)$-dimensional vector of the voltage drops along the sides, $\underline{\Phi}$ is the $N^{2}$-dimensional vector of the absolute potentials at each node, $G$ is a $(B+Q) \times(B+Q)$ square diagonal matrix containing, on its diagonal, the conductances for the sides internal to the mesh, and 0 otherwise. Matrices and vectors involved in the calculations are in general highly dimensional and sparse. Solving the circuit leads to either a quadratical equation or to finding the minimum of a fourth-order polynomial, since both the potential values at the internal nodes and the conductance values of all the resistors are unknowns and they appear under the form of product in the equations. In this paper, we present a method that we call symbolic linear reduction (SLR), where the peculiarities of the system are exploited in order to identify the internal conductances according to a linear algorithm under the working hypothesis that they are different from zero. In this algorithm, the same steps that are used to find the the external conductance values, that we name the external "corona," are also applied to the internal mesh of reduced dimension. The topology of the circuit induces the structure of the matrices involved in the calculations. Thanks to the symbolic solver used, we obtain an explicit expression for the results, simplifying the process of implementing the algorithm in a lower level language. The equations of the external corona are decoupled from the "core," then the same basic laws are applied iteratively until the central conductances are calculated; in this sense, this algorithm can be considered as belonging to the family of the layer-stripping algorithms [4]. This approach has the advantage that all the relevant quantities and processes, such as error propagation, can be calculated for one step only and then derived for the entire network by composition.

\section{Symbolic LineAR REduction ALGORITHM}

In the incidence matrix formalism, rows span over the nodes, whereas columns span over the branches. In order to simplify the decoupling process, the nodes have been numbered in a spiral order from the exterior to the interior of the mesh. For the sake of simplicity again, the branches have been numbered so that the former are relative to the mesh resistors and the latter correspond to the external branches added to inject the currents. The structure of the system then becomes

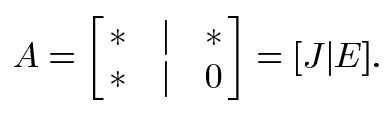

It is interesting to note that, due to the ordering chosen, the left columns of the matrix $A$ concern the internal branches, whereas the right columns refer to the external ones. One can act the same way on the matrix $G$

$$
G=\left[\begin{array}{cc}
D & 0 \\
0 & 0
\end{array}\right] ; \quad \underline{I_{0}}=\left[\begin{array}{c}
0 \\
\widetilde{I}_{0}
\end{array}\right]
$$

where the matrix $D$ is diagonal. Solving the system and using this notation leads from (3)-(5) to the much more compact version

$$
J D J^{T} \underline{\Phi}=\underline{\widetilde{I}_{0}} .
$$

It is worth pointing out that $J$ is still a very sparse matrix whose nonzero entries can only be +1 or -1 and depend only on circuit topology. The diagonal of $D$ contains all the unknown conductances, $\underline{\widetilde{I}}_{0}$ is assigned and thus known. In turn, $\underline{\Phi}$ contains the best estimate one can have of the potential nodes given a set of border measurements, obtained by solving the linear system

$$
E^{T} \underline{\Phi}=\underline{V}^{\mathrm{ext}} .
$$

In an exact arithmetic context, the rank of matrix $E$ enables the explicit calculation of the potentials at the border nodes. In a real case, however, measurements lead to finite precision arithmetic. However, a redundant measurement policy can be adopted, i.e., more measurements are done than the minimal set, and the minimum mean-square error solution can be derived. It is remarkable that the steps derived from the symbolic execution of this algorithm reveal the topology-induced matrix structure and can also be applied when the floating point inputs are used. Equation (9) also tells us that the rank of $E$, which is $4 N-5$, is equal to the maximum number of independent measurements compatible with this type of formalism, which can be performed. Thanks to the ordering of the nodes, the decoupling of the equations of the external corona can be done by simply taking the first rows from the matricial expression represented by (8). Note that it is linear with respect to the conductances present in the matrix $D$. Therefore, (8) can be recast in the following form:

$$
\sum_{a, c} J_{\mathrm{ia}} g_{a} J_{\mathrm{ca}} \Phi_{c}=\widetilde{I}_{0_{i}}
$$

where $i$ spans over the nodes of the corona. This expression is equivalent to

$$
C(\underline{\Phi}) \underline{g}=\underline{\widetilde{I}}_{0} .
$$

One equation of the type (11) is available for each measurement. $g$ is a vector containing the nonnull entries that were previously on the diagonal of $D$ and $C$ is a matrix containing the potential values at the nodes on the external shell. Some of them are known through (9), whereas the innermost need to be determined. All the equations of the form (11) can be collected in one matrix relationship by simple vertical juxtaposition, giving

$$
K(\underline{\Phi}) \underline{g}=\underline{\Gamma}_{0} .
$$

As already mentioned, this equation is quadratic in its unknowns, that is, some potential values and the conductances; however, given its very peculiar structure, it has been possible to solve it through a succession of linear operations, under the unique and realistic assumptions that only nonzero conductances are present. The compact representation $\left[K \Gamma_{0}\right]$ of the system in (12) is composed of many bands, one for each measurement. Matrix (13) is a typical band corresponding to 
a single measurement for the case $N=4$, represented only partially for typographical reasons. $u[i, j]$ are the unknown potential values at the inner border of the corona, which is the border of the core, whereas $\eta[i]$ are values derived by measured border potentials

$\left[\begin{array}{cccccccc}\eta_{1} & \eta_{2} & 0 & 0 & 0 & \ldots & 0 & \eta_{3} \\ 0 & \eta_{4} & \eta_{5}-u_{1_{1}} & \eta_{6} & 0 & \ldots & 0 & \eta_{7} \\ 0 & 0 & 0 & \eta_{8} & \eta_{9}-u_{1_{2}} & \ldots & 0 & 0 \\ 0 & 0 & 0 & 0 & 0 & \ldots & 0 & 0 \\ 0 & 0 & 0 & 0 & 0 & \ldots & 0 & 0 \\ 0 & 0 & 0 & 0 & 0 & \ldots & 0 & 0 \\ 0 & 0 & 0 & 0 & 0 & \ldots & \eta_{20} & 0 \\ 0 & 0 & 0 & 0 & 0 & \ldots & \eta_{23} & 0 \\ 0 & 0 & 0 & 0 & 0 & \ldots & 0 & 0 \\ 0 & 0 & 0 & 0 & 0 & \ldots & 0 & 0 \\ 0 & 0 & 0 & 0 & 0 & \ldots & 0 & 0\end{array}\right]$.

As one can see, there are two kinds of rows: those having three nonzero elements, all of them being a number, and those having four nonzero elements, one of them being a number. The former type corresponds to the nodes located on the corners of the network, whereas the latter corresponds to the corona nodes. Each nonnumeric term contains only one unknown potential value. This structure is induced by the topology of the circuit and is exploited in developing this ad hoc procedure. First, the matrix is ordered in such a way as to gather together all the rows corresponding to the same node. Second, all the full numeric rows are collected into a single submatrix and the derived system is solved yielding the conductances at each network corner. Third, the remaining blocks undergo a transformation that is very similar to the Gauss reduction concerning only the numeric columns; this is done in an order that reduces the trailing terms column at the end of the procedure. These steps transform the matrix in a set of submatrices having the form shown in (14)

$$
\left[\begin{array}{cccc}
u_{3_{1}} a_{1_{2}}+a_{1_{1}} & a_{1_{3}} & \ldots & a_{1_{4}} \\
u_{4_{1}} a_{2_{3}}+a_{2_{1}}+u_{3_{1}} a_{2_{2}} & 0 & \ldots & a_{2_{4}} \\
u_{1_{1}} a_{3_{4}}+a_{3_{1}}+u_{3_{1}} a_{3_{2}}+u_{4_{1}} a_{3_{3}} & 0 & \ldots & 0 \\
u_{7_{1}} a_{4_{3}}+u_{3_{1}} a_{4_{1}}+u_{4_{1}} a_{4_{2}} & 0 & \ldots & 0 \\
u_{8_{1}} a_{5_{3}}+u_{3_{1}} a_{5_{1}}+u_{4_{1}} a_{5_{2}} & 0 & \ldots & 0 \\
u_{9_{1}} a_{6_{3}}+u_{3_{1}} a_{6_{1}}+u_{4_{1}} a_{6_{2}} & 0 & \ldots & 0 \\
u_{2_{1}} a_{7_{3}}+u_{3_{1}} a_{7_{1}}+u_{4_{1}} a_{7_{2}} & 0 & \ldots & 0 \\
u_{10_{1}} a_{8_{3}}+u_{3_{1}} a_{8_{1}}+u_{4_{1}} a_{8_{2}} & 0 & \ldots & 0 \\
u_{11_{1}} a_{9_{3}}+u_{3_{1}} a_{9_{1}}+u_{4_{1}} a_{9_{2}} & 0 & \ldots & 0 \\
u_{5_{1}} a_{10_{3}}+u_{3_{1}} a_{10_{1}}+u_{4_{1}} a_{10_{2}} & 0 & \ldots & 0 \\
u_{6_{1}} a_{11_{3}}+u_{3_{1}} a_{11_{1}}+u_{4_{1}} a_{11_{2}} & 0 & \ldots & 0
\end{array}\right] .
$$

Only the nontrivial columns of the submatrix relative to a single node after processing (case $N=4$ ) are shown, the $a[i, j]$ entries are numeric values, some of them deriving by measured border potentials and some induced by circuit topology. As one can see, most of the rows have only one entry different from zero, which depends on the unknown potential values for the various measurements. Under the assumption that the conductances are nonnull, those entries have to be nullified leading to a set of linear algebraic homogenous systems whose solution provides the potential values at the border of the core. Now, the original linear system, no longer parametric, can be solved and the last unknown conductances of the corona can be derived. Then, the same kind of approach can be applied to the second corona, which is the external corona of the core, and the procedure can go on until the center of the network is reached. A thorough sensitivity analysis for this approach will be an argument of future development; however, the decomposition of a potentially huge system in a succession of smaller and extremely simple linear algebraic systems appears to be of extreme utility.

\section{From SENSOR READING TO POSTURE IDENTIFICATION}

As already mentioned, a very precise knowledge of sensor position and deformation is needed when using a minimal sensor set. Conversely, a redundant sensor set can compensate for inaccuracy due to error propagation intrinsic to the peripheral reading and imperfect knowledge of exact sensor position. Redundancy is, however, at the expense of a more complex way to interpret the readings. This issue has been faced by using a suitable "inversion" technique that allows mapping the reading space to the posture space. Let us suppose that sensors have a monotonic stress versus resistance characteristics and that their location is appropriate, which means that at least one of them is actually affected by any relevant movement of the subject. First, let us make some considerations on what the determination of human posture means and how sensor networks can be employed. To define a posture formally, it is necessary to develop a physical model for the particular subject holding it. We attribute a certain number of cartesian frames, one for each considered degree of freedom. In this sense, a posture is simply the set of the mutual positions with respect to the fixed frames. Obviously, the entire set of the mutual positions is not necessary to reconstruct a posture exactly, and a minimal set can be chosen in many different ways. The Denavit-Hartemberg algorithm [12], for example, fixes exactly the number of relations between frames and gives a standard method to write these positions in terms of rotation and translation affinities, for rotational and translational joints. In the case that the topological structure of the kinematic chain under study cannot be linearly approximated, it is still possible to define a model by using more sophisticated nonlinear approaches to describe the kinematics more accurately [13]. The problem can be formalized as follows. Let us assume a fixed-state space (described by a set of frames assigned and by their mutual coordinate transformations) which we will designate as the posture space $\Theta$ and which admits a well-defined topological model. To survey posture, it is necessary to construct a metric on this space and then to relate the elements of $\Theta$ to the electrical sensor configurations that span the space $S$ of sensor readings. The sensors are positioned with a certain topology. It is assumed that they are able to detect a variation in the subject's posture and that there exists an invertible function $\mathbf{F}$ that maps the space of the postures into $S$. As a consequence, the image of $\Theta$ through $\mathbf{F}$ is a subset of $S$ which has the same dimension of $\Theta$ itself. Therefore, the inverse of $\mathbf{F}$ can be used to infer the posture from the electrical readings. The construction of $\mathbf{F}$, otherwise named "system identification," is the crucial point of the method, and it is important from several points of view. It is worth pointing out that this phase is not a single-sensor calibration, but a real identification of the entire 
system. In fact, for several reasons (the most important being the variability of body structure of the subject), the sensor location is not precisely known. However, adopting the described approach, this requirement is no longer ineludible, neither is the map relating the size of a particular sensor to its electrical resistance. To better explain this point, one should consider that adherence of a sensorized fabric to the subject gives rise to intrinsic crosstalk phenomena, due to the nature of the textile on which sensor are positioned. This fact, instead of being an inconvenience, is instrumental to the method we have developed and ensures the possibility of reconstructing posture without the knowledge of the exact location of every single sensor. The identification concerns not only the set of sensors, but also the body structure of the subject. The same garment shown in Fig. 1(a) can then, in principle, be used to detect the posture of many different subjects with the prescribed accuracy, shifting all the variability on a different function $\mathbf{F}$. Metric introduction in the space of postures is realized simultaneously with the construction of F. The basic idea is to relate the information originating from a conventional measurement system (set of electrogoniometers, optical tracking system) to the electrical state of a set of sensors. The former is obtained for a set of postures suitably chosen according to the topological structure of $\Theta$. This care is necessary because the space $\Theta$, related to anatomical variables such as bones and joint positions, is not directly accessible to the observer

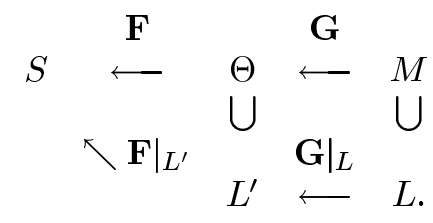

Then, a third space $M$ (called "space of the markers") is introduced with its own coordinate frame, endowed with the same topology of $\Theta$ and of the property of effectivity, meaning that it is always possible to determine whether two points in $M$ coincide or not. Let us call $\mathbf{G}$ the continuous bijective function mapping $\Theta$ into $M$. Due to the effectiveness property, $M$ has the advantage, with respect to $\Theta$, of being directly accessible. Let us now fix a system of coordinates and a metric in $M$, if $\mathbf{G}$ is differentiable, a metric is automatically induced also on $\Theta$. If now a lattice $L$, that is a finite discrete set of points, is chosen in $M$, then another lattice $L^{\prime}$ in $\Theta$ is uniquely identified via the map $\mathbf{G}$, and a metric is induced accordingly. Determining the law of correspondence between the lattice $L$ and its image in the space $S$ (which is known by acquiring the values presented by the set of sensors when a position of $L$ is held fixed) means knowing the restriction of $\mathbf{F}$ on $L^{\prime}$. The next step is to expand the knowledge of $\mathbf{F}$ from this restriction to all $\Theta$, obtaining $\mathbf{F}$. This is a problem of multivariate interpolation that will be discussed in the next section.

\section{INTERPOLATION TECHNIQUE}

In order to reconstruct positions and movements not included in $L^{\prime}$, a multivariate interpolation of $\mathbf{F}$ component by component has been employed. To approach this problem a piecewise linear (PL) interpolation can be performed, either directly on
$\mathbf{F}$ or on its inverse. We choose the direct approach because of complexity issues that will be clarified in the following. In the direct case, we want to determine a class of linear applications from $\Theta \rightarrow R$, each one holding on a certain subset of $\Theta$. The union of these subsets must contain $\Theta$ and the functions corresponding to adjacent subsets must coincide on their intersection. Due to the analyticity of linear applications, it is clear that two of these subsets can intersect only at their borders. The more time-consuming part of the algorithm is the partitioning of an $n$ hypercube whose vertices belong to $L^{\prime}$. In order to solve the linear problem of interpolating a function defined on a given lattice (assumed to be, i.e., homeomorphic to $Z^{n}$ (where $n$ is the dimension of the space of the posture), a partitioning in $O(n !)$ hyper-tetrahedra is necessary (a proof that this partitioning exists and is minimal can be given by induction on the dimension of $\Theta$ ). Using this partitioning, the interpolation is given by finding $O(n !)$ hyperplanes passing each one through $n+1$ points, representing the vertices of a hyper-tetrahedron. The algorithm has an exponential complexity in the dimension of $\Theta$, and this is the reason for the direct approach option. The last step consists of mapping the measured sensor values into a posture by means of the interpolated function. Two ways of doing this have been considered on line and off line. In the on-line method, the function $\mathbf{F}$ is, at first, interpolated on a finer lattice; then, during the signal acquisition, each component of $\mathbf{F}$ is set to be equal to the corresponding sensor measurement. Solving this system onto the lattice gives the posture; thus, this must be done for each reading and requires noticeable computational resources to be performed in real time. This method is exact, but for higher dimensional phase spaces, it must be replaced by some iterative algorithm minimizing the discrepancy between the predicted and the actual postures. The off-line method estimates a pseudoinverse of the function $\mathbf{F}$, which is done only once, and then applies it to every measurement. This method is time consuming when it calculates the $O(n !)$ pseudoinverses of the pieces approximating $\mathbf{F}$. When the number of the variables involved becomes high, a more suitable tool to perform this task is an artificial neural network (ANN). It can be trained to fit the presented postures using the sensor readings as input. The cost function, which is the square difference between the postures, which belong to $\Theta$, and the output of the ANN, can be minimized via a nonlinear programming algorithm, such as conjugate gradient. As is typical with ANNs, the training phase can take some time, but the forward phase is very fast.

\section{EXAMPLE OF METHODOLOGY IMPLEMENTATION: THE UPPER LIMB POSTURE DETECTION}

The methodology developed in Sections VI and VII has been employed to implement several prototypes of sensing garments able to detect the posture of diverse segments of human kinematic chains. Here, we present an example of an upper-limb kinesthetic interface. We monitor two joints of the upper limb: the gleno-humeral joint and the elbow joint.

\section{A. Definition of the Physical Model}

In the shoulder, the gleno-humeral joint is a ball-and-socket joint formed by the humeral head mating with the opposing 
glenoid cavity of the scapula. Both the joint surfaces can be represented as spherical segments, but the different curvatures induce an intrinsic nonlinearity in the geometry of the configurational space [14]. Locally, the gleno-humeral joint is a three DOFs system that we designate as usual in kinesiology: adduction-abduction, flexion-extension (angulation in a frontal and in a sagittal plane respectively), and rotation. Globally, a combination of flexion, adduction, extension, and abduction produces rotations that explain the global entanglement of the DOFs [14]. The elbow is a composite joint where two convex articular surfaces at the distal extremity of the humerus mate with two concave surfaces at the proximal extremities of the radius and the ulna (humero-radial and humero-ulnar joints). Furthermore, the convex circumference of the radial head mates with the concave radial notch of the ulna forming a third articulating territory (proximal radio-ulnar joint). Displacement is allowed for two DOFs, namely elbow flexion-extension and forearm pronation-supination. Intrinsically, nonlinear minor phenomena of the configurational space are neglected. Let us consider four different systems of coordinates:

1) $T_{0}$ (said absolute frame) centered in $O_{0}$ at the bottom of a subject in standing position, with the $z$ axis along the vertical axis of the subject, the $y$ axis along the transversal axis, and the $x$ axis along the sagittal axis (for the definitions of position and anatomical planes and axes see [15]);

2) $T_{1}$, fixed and parallel with respect to $T_{0}$, but centered in the center of rotation of the humerus head $\left(O_{1}\right)$;

3) $\mathrm{T}_{2}$, fixed on the distal extremities of the humerus, $\mathrm{O}_{2}$ with the $x$ axis parallel to the geometric axis [14] of the humerus and the $z$ axis parallel to the axis of the rotation of radio and ulna, which constitutes the main part of the movement of flexo-extension;

4) $T_{3}$, fixed on the wrist $\left(O_{3}\right)$, and oriented as $T_{2}$ when the anatomical position is held by the subject. A complete representation of the defined frames and of the Denavit-Hartemberg parameters is provided in Fig. 7(a).

By writing the affinities that transform the coordinates between the given frames, it is possible to express the positions of the points $O_{1}, O_{2}$, and $O_{3}$ in the frame $T_{0}$ in terms of the Denavit-Hartemberg parameters $\theta_{1}, \theta_{2}, \theta_{3}, \theta_{4}$, and $\theta_{5}$, which physically represent, respectively, the adduction-abduction angle, the flexion-extension angle, and the rotation angle for the shoulder and the flexion-extension angle and pronation-supination angle for the elbow. The set of fixed frames and the affinities constitute the model for the kinematic chain.

\section{B. Definition of $M$ Space}

We now have to define the space $M$ of the markers and the application $\mathbf{G}: \Theta \rightarrow M$. Let us consider a subject wearing the garment and standing up in the center of a certain bounded environment (that we suppose cubic, with no loss of generality). Let $W$ be the internal surface coinciding with the walls of the cube and let $M=W^{4}$, where $\Theta$ can be embedded, as will be clarified by the construction of $G$. In order to define $\mathbf{G}$, let us consider the straight line $r_{1}=\left\{x=t O_{3}+(1-t) O_{2}, t \in R\right\}$ passing through $O_{3}$ and $O_{2}$ (parallel to the axis of the forearm),
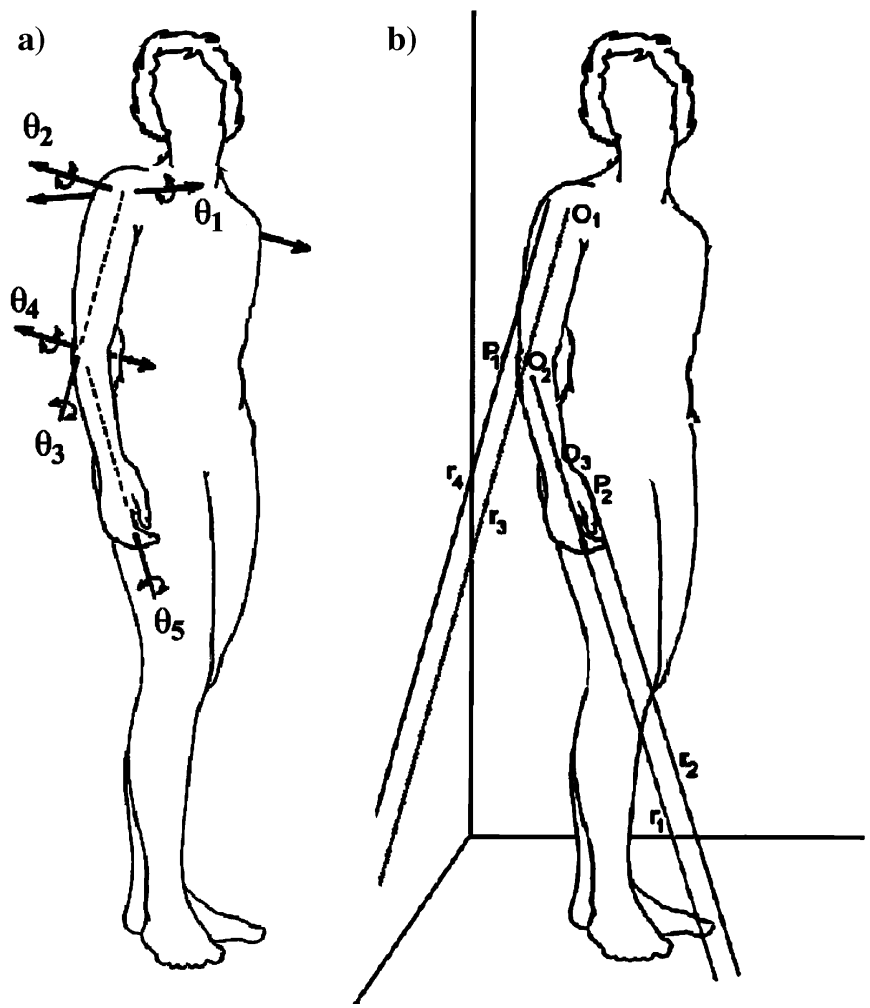

Fig. 7. (a) Axes of rotation of the movements. (b) Construction of $\left(M,\left.\mathbf{F}\right|_{L^{\prime}}\right)$. The set of the markers is defined by the intersections of the straight lines $r_{1}$, $r_{2}, r_{3}$, and $r_{4}$ with the walls of the environment, while $\left.\mathbf{F}\right|_{L^{\prime}}$ is the change of coordinates from $L^{\prime}$ to $M$.

the line $r_{2}=\left\{x=t\left(O_{3}-O_{2}\right)+P_{1}, t \in R\right\}$ parallel to the first one and lying in a plane containing the palm of the hand, a straight line $r_{3}=\left\{x=t O_{2}+(1-t) O_{1}, t \in R\right\}$ passing for $O_{2}$ and $O_{1}$ a straight line $r_{4}=\left\{x=t\left(O_{2}-O_{1}\right)+P_{2}, t \in R\right\}$ parallel to $r_{3}$ fixed on the humerus and passing through $P_{2}$ [see Fig. 7(b)]. Clearly, these four lines intersect the planes representing the walls of the cube in eight points (except in some trivial singularities) and it is easy to prove that the map $\mathbf{G}$ that relates the position of the subject to four of these intersections (chosen on different lines) is invertible. Fixing a subset $L^{\prime} \subset \Theta$ by the computation of the intersections explained above, $L$ is completely determined. In Fig. 8, a subset of $L$ is drawn on the wall of the environment. The subject under measurement points to each element of $L$. By reading the sensor resistances, it is possible to build the discrete application that maps $L$ to $S$. By combining this application with $\mathbf{G}$, the restriction of $\mathbf{F}$, to $L^{\prime}$, namely $\mathbf{F}^{\prime}$, is completely determined. The next task is to expand this discrete map to an interpolation of $\mathbf{F}$.

\section{Signal Acquisition and Interpolation}

In order to reconstruct positions not included in $L^{\prime}$, the PL multivariate interpolation of the discrete map obtained in the previous paragraph was employed, as described in the theory, Section VII. In this particular example, the on-line version is implemented. To obtain the data necessary for the construction of $\mathbf{F}^{\prime}$, the sensor values corresponding to points in $L^{\prime}$ have been acquired. The device used consists of a general purpose National Instruments data and a subsequent ad hoc buffer and filter $(0 \div$ 8-Hz low pass). After their conversion to a 8-bit format, data 


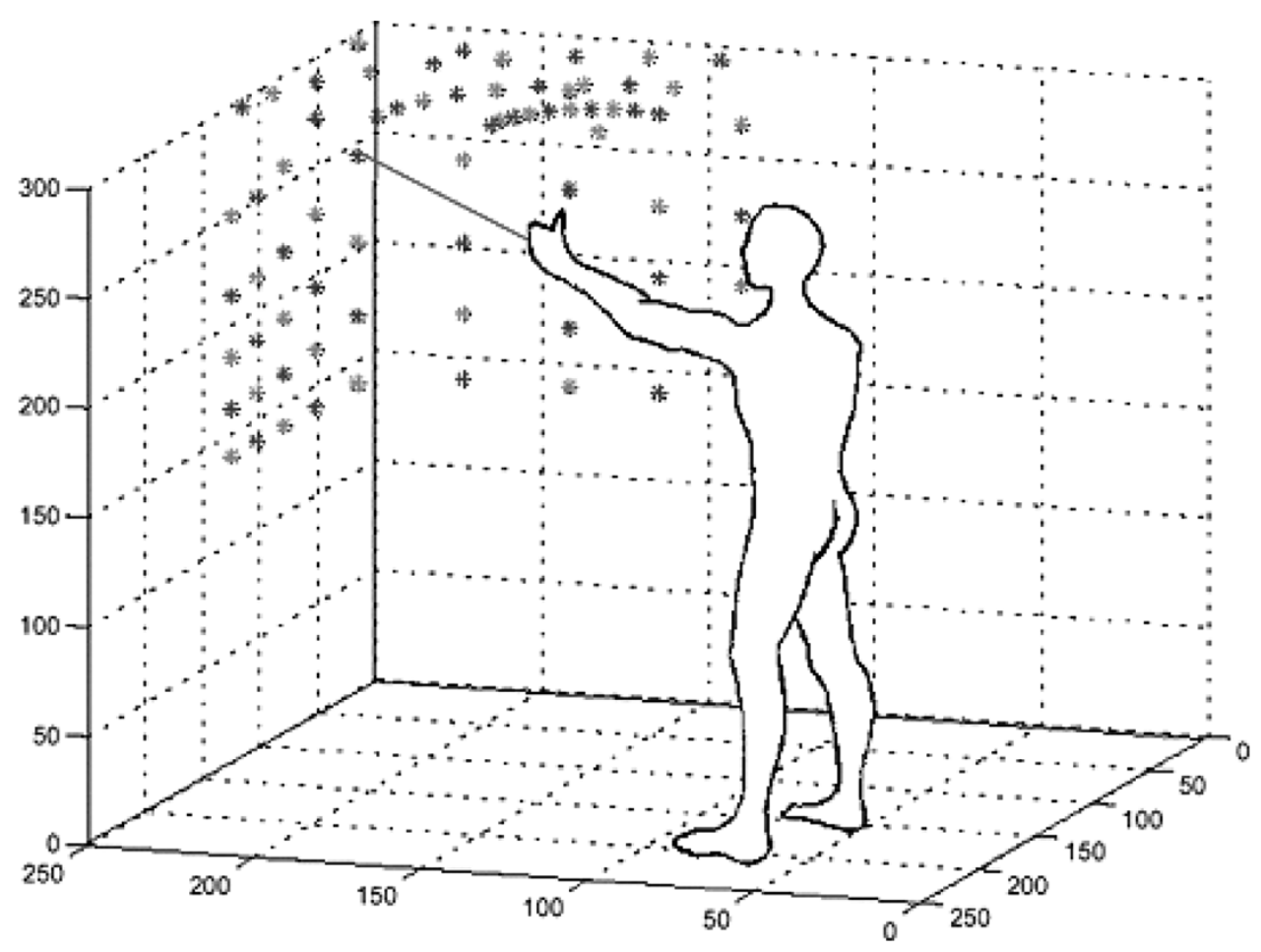

Fig. 8. Construction of $L^{\prime}$ and $\mathbf{F}^{\prime}$. In order to calibrate the system, the subject points at different dots in $M$ while the corresponding sensor resistance values are read.

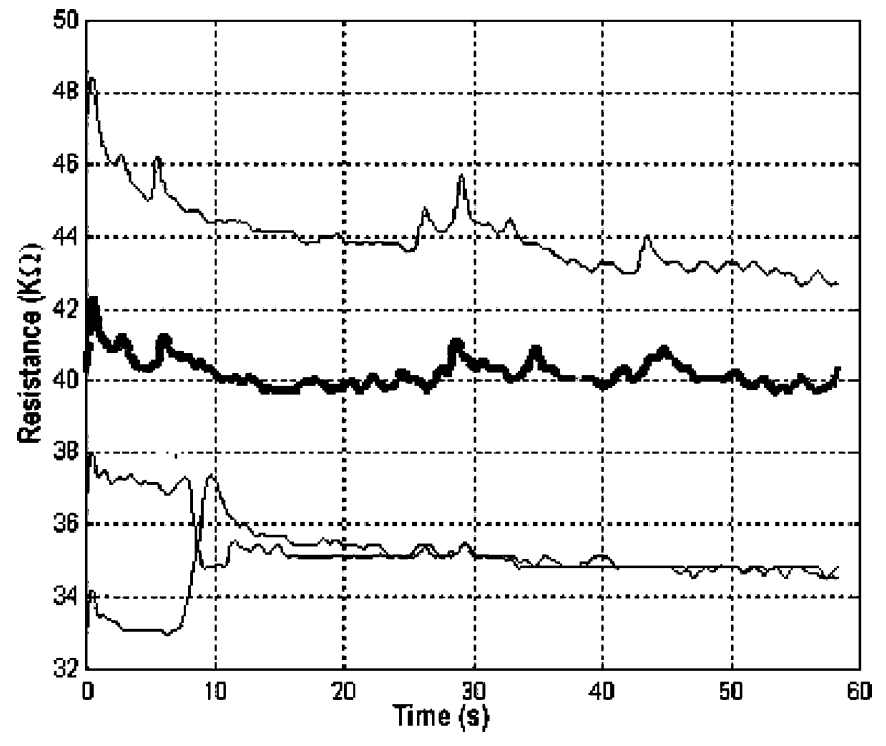

Fig. 9. Sensor signals deriving from garment (versus time) after their A/D conversion.

became available in a buffer to be directly read by the acquisition and interpolation software. An on-purpose visualization form has been developed and dedicated to check the quality of the signals after their digital conversion, as reported in Fig. 9.

In order to avoid the cumbersome wiring coming from a more dense readout, data can be sent to a central unit via a telemetric device. We are presently moving in this direction. The prototypes we have realized consist in a an electronic unit based on a microprocessor able to acquire and convert in digital format the signals coming from fabric sensors, and send them via radio frequency to a remote monitoring station.

\section{RESULTS}

The application of our methodology provided quite an accurate reconstruction of the posture of the arm in a subject wearing the adherent sensorized garment in which information on sensor location was inaccurate. Moreover, this technique has shown good capabilities in balancing the irregularities of operation of individual sensors. In order to evaluate the precision of the method used, we have chosen a subspace $\tilde{L} \neq L^{\prime}$ (named subspace of the targets) contained in $L$ and we have acquired the sensor values corresponding to the points of $\tilde{L}$, without using them in the construction of $\mathbf{G}$. In this way, it has been possible to reconstruct the (known) positions of points in $\tilde{L}$ by using the inverse of $\mathbf{F}$ and to evaluate the error introduced by the method.

Despite the intrinsic difficulty in representing high-dimensional quantities in a synthetic manner, we tried to express the performance of the method by showing a simple posture reconstruction on bidimensional charts where the angles $\theta_{1}, \ldots, \theta_{5}$ are represented. After reading the sensors, the surfaces corresponding to the solution of $F_{i}\left(\theta_{1}, \ldots, \theta_{n}\right)=s_{i}$ for each component $i$ of $\mathbf{F}$ are generated, being $s_{i}$ is the $i t h$ sensor reading. In Figs. 10 and 11, the intersections of these manifolds with three meaningful planes are shown for the sake of representation simplicity. These planes are obtained keeping fixed three out of the five studied angles. In the on-line interpolation method (see Section VII), these manifolds are subspaces of a lattice; therefore, they are discrete and it is very likely that they do not intersect at any point. Because of that, we devised a criterion that minimizes the distance between points belonging to different manifolds in order to estimate the target location. According to the number of sensors in this example, there are eight of these manifolds; 

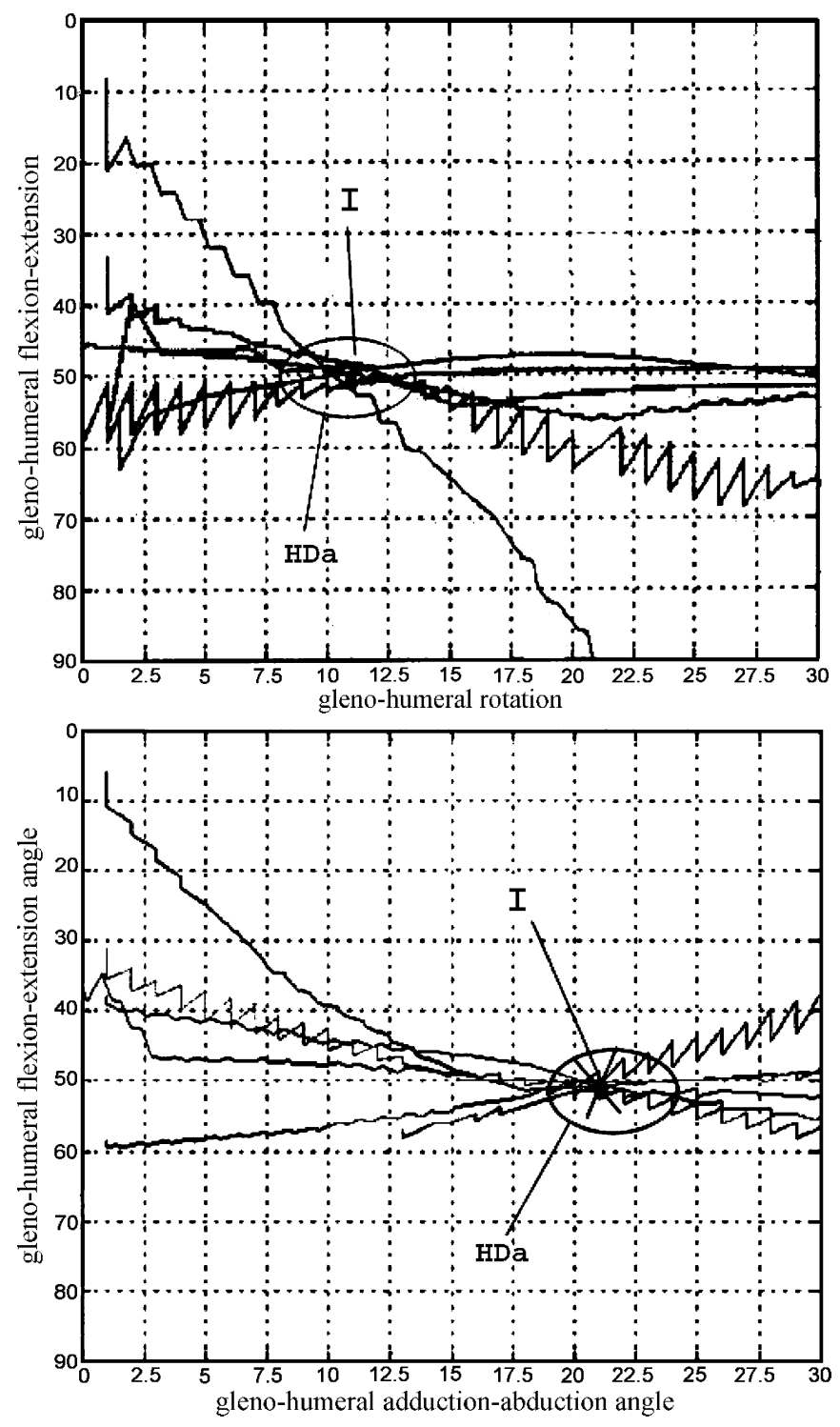

Fig. 10. Posture detection for the shoulder: A position is determined by the intersection of the manifold on which sensors hold the values read by the garments. "I" represents the real target we used to test the system and $\mathrm{HDa}$ represents the high-density area of the solution of all the $F_{i}\left(\theta_{1}, \ldots, \theta_{n}\right)=s_{i}$. Only $\theta_{1}, \theta_{2}$, and $\theta_{3}$ are shown here.

the distance between the calculated position and the real one is roughly $10 \mathrm{~cm}$, which is a relative error of $4 \%$. The reconstruction presented here shows the methodology used. Table II summarizes the other experiments we did to test the approach.

The estimation error obtained considering all the experiments done never exceeded $8 \%$.

\section{SUMmary AND CONCLUSIONS}

Fabrics coated with conducting polymers as PPy or a mixture of carbon and rubber have piezoresistive properties and they can be used to realize strain sensors, with useful applications in the field of man-machine interfaces. In particular, we have shown that these fabrics can be easily integrated into truly wearable, instrumented garments, capable of recording kinaesthetic maps of human motor function with no discomfort for the subject. Besides, a method was presented allowing to operate with large

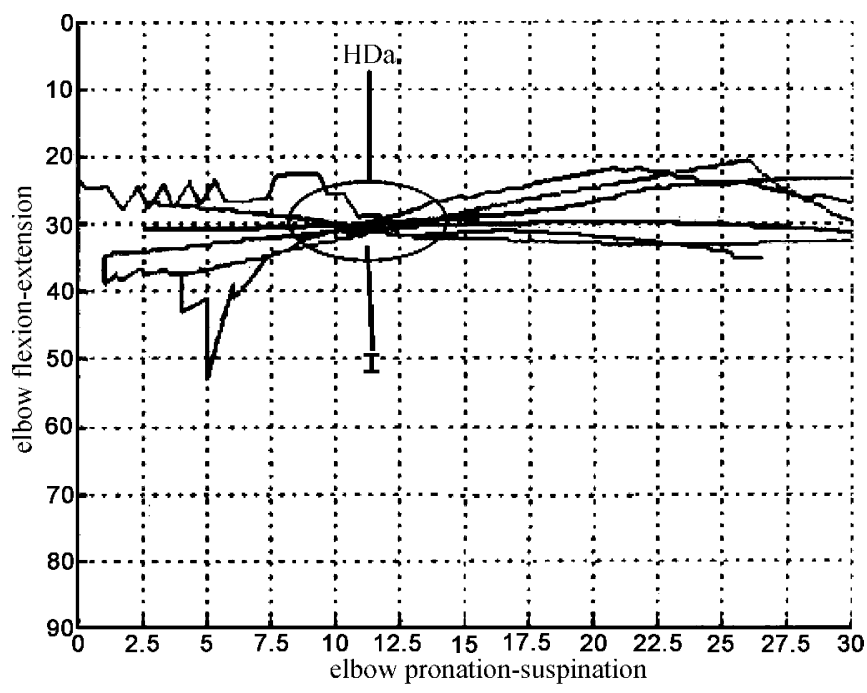

Fig. 11. Posture detection for the elbow.

TABLE II

Sample Measurements of Shoulder and Elbow Posture

\begin{tabular}{l|c|c|c|c|c}
\hline & $\theta_{1}$ & $\theta_{2}$ & $\theta_{3}$ & $\theta_{4}$ & $\theta_{5}$ \\
\hline real position & $30.0^{\circ}$ & $20.0^{\circ}$ & $20.0^{\circ}$ & $11.0^{\circ}$ & $30.0^{\circ}$ \\
est. position & $31.4^{\circ}$ & $22.6^{\circ}$ & $25.5^{\circ}$ & $12.5^{\circ}$ & $32.0^{\circ}$ \\
real position & $30.0^{\circ}$ & $80.0^{\circ}$ & $20.0^{\circ}$ & $1.0^{\circ}$ & $50.0^{\circ}$ \\
est. position & $33.2^{\circ}$ & $78.6^{\circ}$ & $24.3^{\circ}$ & $1.2^{\circ}$ & $53.4^{\circ}$ \\
real position & $10.0^{\circ}$ & $0.0^{\circ}$ & $20.0^{\circ}$ & $11.0^{\circ}$ & $50.0^{\circ}$ \\
est. position & $9.2^{\circ}$ & $3.1^{\circ}$ & $2.4^{\circ}$ & $12.2^{\circ}$ & $49.3^{\circ}$ \\
real position & $70.0^{\circ}$ & $80.0^{\circ}$ & $70.0^{\circ}$ & $21.0^{\circ}$ & $50.0^{\circ}$ \\
est. position & $74.0^{\circ}$ & $83.5^{\circ}$ & $72.6^{\circ}$ & $22.5^{\circ}$ & $52.0^{\circ}$ \\
\hline
\end{tabular}

sets of sensors positioned on garments without dramatically increasing the number of reading channels. Various connection topologies have been analyzed and a novel algorithm for acquisition has been described in detail. From an abstract point of view, these sets of sensing fabric patches linked in different topological networks can be regarded as a spatially distributed sensing field. By simultaneously comparing the sensing field with the value of the joint variables in the identification phase, it is possible to reconstruct postures in the data acquisition phase. The analysis exposed in this paper guarantees that all the information needed for the reconstruction can be effectively gathered. It also provides a key understanding of the single-sensor influence, as well as strategies for density and location of the sensors. Additionally, we have studied a reconstruction (inversion) technique based on an identification phase of the entire system. In fact, it is worth noting that, in the scope of a synthetic and real-time data processing, one can avoid passing through the sensor space and consider all the sensors as a unique entity to be read and interpreted. This has the further advantage of making the hardware applicable, regardless of the specific body structure. In order to assess the fundamental limitations of the method, we are testing it over large sensor networks to assay its real-time behavior. Besides, a delicate point is represented by the sensitivity of the approach and how it depends on the precision of the measurements. The emerging theme of this paper is that, while for many applications, the actual local information is desirable, in many others, a holistic view is more suitable. 


\section{APPENDIX}

Let us consider a square mesh network, as reported in Section IV. The aim of this appendix is to prove that it is possible to distinguish two resistance networks with the same topology, but different resistance values only by reading the current values flowing into the network, by imposing fixed voltages at the borders.

With reference to the network in Fig. 6, let EN be the set of the external nodes of the network

$$
\begin{aligned}
\mathrm{EN}=\{(1,1),(1,2) & \ldots(1, \mathrm{~N}),(2,1),(2, \mathrm{~N}), \\
& \ldots(N-1,1),(N-1, N),(N, 1),(N, N)\}
\end{aligned}
$$

and let $>$ to be a total ordering on EN. Let us consider the subset of $\mathrm{EC} \subset \mathrm{EN} \times \mathrm{EN}$ (external couple) defined by

$$
\mathrm{EC}=\left\{\left(N_{1}, N_{2}\right) \in \mathrm{EN} \times \mathrm{EN} \mid N_{1}>N_{2}\right\}
$$

We prove in the following that, by supplying the network through each element of EC and by reading the currents that enter the circuit, it is possible to distinguish two networks with the same topology but different resistance values. With no loss of generality, it is possible to suppose that a unitary voltage is applied to the circuit (due to the linearity of the network) and that two networks differ only for resistances inside in (otherwise, we can always add a new set of known fixed resistance to form a new border and consider a new network with $n+2$ nodes on its side). Let us suppose to supply the network through a certain element $k \in$ EC. By writing the equation of the circuit (by the current mesh method), we obtain

$$
R_{k}^{*} I_{k}^{*}=e_{m+1}
$$

where $m=(N-1)^{2}$ is the number of the passive mesh, $e_{m+1}$ is an element of the canonical bases of $\mathbf{R}^{m+1}, R^{*}$ the matrix of the resistance, and $I^{*}$ the vector of the currents. Equation (15) can be written as

$$
\left[\begin{array}{cc}
R & b_{k} \\
b_{k}^{T} & c_{k}
\end{array}\right]\left[\begin{array}{c}
I^{k} \\
I_{\mathrm{ext}}^{k}
\end{array}\right]=\left[\begin{array}{l}
0 \\
1
\end{array}\right]
$$

Let us observe that $R_{k}^{*}$ changes only in its last row and its last column, because of the network changes in the different measurement only for it supplied mesh, while the set of passive mesh remains the same.

It is easy to show that $R$ is a block-tridiagonal matrix of the form

$$
\left[\begin{array}{ccccc}
T_{1} & J_{2,1} & \ldots & 0 & 0 \\
J_{1,2} & \cdots & \ldots & 0 & 0 \\
\ldots & \ldots & \ldots & \ldots & \cdots \\
0 & 0 & \ldots & \ldots & J_{N-1, N} \\
0 & 0 & \ldots & J_{N, N-1} & T_{N}
\end{array}\right]
$$

where the blocks $J_{i, j}$ are diagonal

$$
\left[\begin{array}{ccccc}
-R_{i, j, 1} & 0 & \ldots & 0 & 0 \\
0 & \ldots & \ldots & 0 & 0 \\
\ldots & \ldots & \ldots & \ldots & \ldots \\
0 & 0 & \ldots & \ldots & 0 \\
0 & 0 & \ldots & 0 & -R_{i, j, N}
\end{array}\right]
$$

and the blocks $T_{i}$ are in the form

$$
\left[\begin{array}{ccccc}
* & -R_{i, j, 1} & \ldots & 0 & 0 \\
-R_{j, i, 1} & \ldots & \ldots & 0 & 0 \\
\ldots & \ldots & \ldots & \ldots & \ldots \\
0 & 0 & \ldots & \ldots & -R_{i, j, N-1} \\
0 & 0 & \ldots & -R_{j, i, N-1} & *
\end{array}\right] .
$$

Briefly, the matrix (17) has dimension $(N-1)^{2} \times(N-$ $1)^{2}$ and is built as follows. Each element on its diagonal $(*)$ represents a certain mesh of the network and contains the sum of the resistances of the same mesh. Each nondiagonal element, individuated by a couple of index $(i, j)$ is equal to the resistance common to the $i$ th and the $j$ th mesh with negative sign. Each resistance in the net occurs almost once or in a matrix $T_{i}$ or in a matrix $J_{i, j}$ as an isolated element. It is easy to prove, by using the Gershgorin theorem [16], that the matrix $R$ is nonsingular. Equation (16) can be split into

$$
\begin{aligned}
R I^{k}+I_{\mathrm{ext}}^{k} b_{k} & =0 \\
b_{k}^{T} I^{k}+c_{k} I_{\mathrm{ext}}^{k} & =1
\end{aligned}
$$

by replacing (20) into (21) and solving with respect to $I_{\text {ext }}$, we obtain

$$
I_{\mathrm{ext}}^{k}=\frac{1}{c_{k}-b_{k}^{T} R^{-1} b_{k}} .
$$

If we consider all the measurements for $k \in \mathrm{EC}$, an expression of (22) would be computable, we could prove that the set $\left.\left\{I_{\text {ext }}^{k}\right\}\right|_{k \in \mathrm{EC}}$, as set of functions of the internal resistances of the network, is independent. Unfortunately, symbolic inversion of the matrix $R$ has high computational costs, and to compute its inverse is very hard for $N=4$ already. What we can do is prove that for two different networks, represented by the matrix $R_{1}$ and $R_{2}$, respectively, it is possible to to find out if they are the same or not.

Let us consider the (20) and (21) for two different networks

$$
\begin{aligned}
& R_{j} I_{j}^{k}+I_{\mathrm{ext}, j}^{k} b_{k}=0 \\
& b_{k}^{T} I_{j}^{k}+c_{k} I_{\mathrm{ext}, j}^{k}=1
\end{aligned}
$$

where $j=1,2$. Let us observe that $I_{\mathrm{ext}, 1}^{k}=I_{\mathrm{ext}, 2}^{k}=I_{\mathrm{ext}}^{k}$; otherwise, the networks would be distinguishable. We can write

$$
\begin{aligned}
& R_{j} I_{j}^{k}+I_{\mathrm{ext}}^{k} b_{k}=0 \\
& b_{k}^{T} I_{j}^{k}+c_{k} I_{\mathrm{ext}}^{k}=1
\end{aligned}
$$

and by combining

$$
c_{k}-b_{k}^{T} R_{j}^{-1} b_{k}=\frac{1}{I_{\mathrm{ext}}^{k}} .
$$


By subtracting (27) evaluated for $j=2$ from (27) evaluated for $j=1$, we obtain

$$
b_{k}^{T}\left(R_{1}^{-1}-R_{2}^{-1}\right) b_{k}=0 .
$$

Due to the univocity of the inverse, it is clear that if it were $R_{1}^{-1}-R_{2}^{-1}=0$, then $R_{1}=R_{2}$. But this condition, for the structure of the blocks $T_{i}$ and $J_{i, j}$, implies that all the resistances of the networks were the same. Let us consider (28) again. $b_{k}$ is the vector that represents the resistance involved in the supplied mesh that changes when $k$ spans EC. It is easy to prove that $\left.\left\{b_{k_{1}} \otimes b_{k_{2}}\right\}\right|_{k_{1}, k_{2} \in \mathrm{CE}}$ spans a set of generators for $R^{N \times N}$, and for the bilinearity of $b_{k_{1}}^{T}\left(R_{1}^{-1}-R_{2}^{-1}\right) b_{k_{2}}$ with respect to $b_{k_{1}}$ and $b_{k_{2}}$, it implies that from (28) results

$$
R_{1}^{-1}-R_{2}^{-1}=0
$$

that what we wanted to prove.

\section{ACKNOWLEDGMENT}

The authors would like to thank Smartex s.r.l., particularly Dr. R. Paradiso, for the material provided.

\section{REFERENCES}

[1] V. J. Lumelsky, M. S. Shur, and S. Wagner, "Sensitive skin," IEEE Sensors J., vol. 1, pp. 41-51, June 2001

[2] D. De Rossi, A. Della Santa, and A. Mazzoldi, "Dressware: Wearable hardware," Mater. Sci. Eng. C, vol. 7, pp. 31-35, 1999.

[3] M. Cheney, D. Isaacson, J. C. Newell, S. Simske, and J. Goble, "NOSER: An algorithm for solving the inverse conductivity problem," Int. J. Imag. Syst. Technol., vol. 2, pp. 66-75, 1990.

[4] M. Cheney, D. Isaacson, and J. C. Newell, "Electrical impedance tomography," SIAM Rev., vol. 41, no. 1, pp. 85-101, 1999.

[5] F. J. Clark and K. W. Hork, "Kinesthesia," in Handbook of Perception and Human Performance USA, K. R. Roff, L. Kaufman, and J. P. Thomas, Eds. New York: Wiley, 1986, vol. I, ch. 13, pp. 1-62.

[6] D. De Rossi, F. Lorussi, A. Mazzoldi, P. Orsini, and E. P. Scilingo, "Active dressware: wearable kinesthetic systems," in Sensors and Sensing in Biology and Engineering, E. P. Barth, E. P. Humphrey, and E. P. Secomb, Eds. New York: Springer-Verlag.

[7] R. V. Gregory, W. C. Kimbrell, and H. H. Kuhn, "Electrically conductive nonmetallic textile coatings," J. Coated Fabrics, vol. 20, Jan 1991.

[8] E. P. Scilingo, F. Lorussi, A. Mazzoldi, and D. De Rossi, "Strain sensing fabrics for wearable kinaesthetic-like systems," IEEE Sensors J., vol. 3, pp. 460-467, Aug. 2003.

[9] D. De Rossi, F. Carpi, F. Lorussi, R. Paradiso, E. P. Scilingo, and A. Tognetti, "Electroactive fabrics and wearable man-machine interfaces," in Wearable Electronics and Photonics, X.-M. Tao, Ed. Cambridge, U.K.: Woodhead.

[10] E. R. Post, M. Orth, P. R. Russo, and N. Gershenfeld, "E-broidery: Design and fabrication of textile-based computing," IBM Syst. J., vol. 39, no. $3 / 4,2000$.

[11] E. Bonderover and S. Wagner, "Transistor fibers for e-textiles amorphous silicon thin film transistors on kapton fibers," in Proc. Int. Interactive Textiles for the Warrior Conf., Boston, MA, July 2002.

[12] J. Denavit and R. S. Hartenberg, "A kinematic notation for lower-pair mechanism based on matrices," J. Appl. Mech., pp. 215-221, June 1955.

[13] D. J. Montana, "The kinematics of contact and grasp," Int. J. Robot. Res., vol. 7, no. 3, pp. 17-32, 1988.

[14] M. A. McConnail and J. V. Basmajian, Muscles and Movements. New York: Krieger, 1977.

[15] I. A. Kapandji, "Physiologie articulaire. Schémas commentes de méchanique humaine," Tome 1: Membre Supérieur, 1999.

[16] A. Quarteroni, R. Sacco, and F. Saleri, Numerical Mathematics. New York: Springer, 2000.

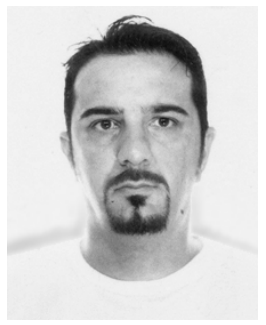

Federico Lorussi received the degree in mathematics from the University of Pisa, Pisa, Italy, in 1999 and the Ph.D. degree in robotics and bioengineering from the University of Pisa in 2003.

Currently, he is with the Information Engineering Department, University of Pisa. His scientific activities are related to anthropomorphic robotics and biomechanics. He is the author of several papers, contributions to international conferences, and chapters in international books

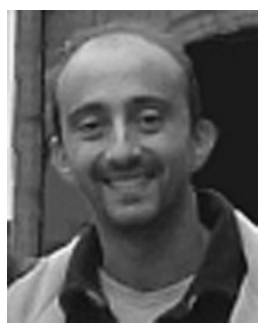

Walter Rocchia received the degree (cum laude) in electronic engineering from the University of Genoa, Genoa, Italy, in July 1996, with a thesis on quantum computation, and the Ph.D. degree in electronic devices from the University of Trento, Trento, Italy, in 2000 , concerning chips based on quanto-mechanical laws.

He was a Research Scholar with the Biochemistry Department, Columbia University, New York, working on the calculation of the electrostatic field generated by biological macromolecules. Then, he spent time at the Corporate Technology Center, Honeywell International, working on carbon nanotube physical properties. He was also a Consultant for the Research Center E. Piaggio, University of Pisa, Pisa, Italy, modeling physical properties of actuating polymers. He is currently with Nest-INFM, Scuola Normale Superiore, Pisa, working on biophysical molecular modeling.

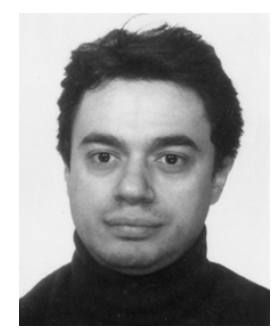

Enzo Pasquale Scilingo received the Laurea degree in electronic engineering from the University of Pisa, Pisa, Italy, and the Ph.D. degree in bioengineering from the University of Milan, Milan, Italy, in 1995 and 1998, respectively.

For two years, he was Postdoctoral Fellow with the Italian National Research Council and he is currently a Postdoctoral Fellow at the Information Engineering Department, University of Pisa, where he pursues his research work at the Interdepartmental Research Center E. Piaggio. He is the author of several papers and contributions to international conferences and chapters in international books. His research interests are in haptic interfaces, biomedical and biomechanical signal processing, modeling, control, and instrumentation.

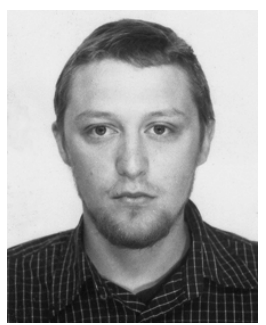

Alessandro Tognetti was born in Lucca, Italy, in 1975. He received the Laurea degree in electronic engineering from the University of Pisa, Pisa, Italy, in April 2001. His thesis was on carbon nanotube actuators. He is currently pursuing the Ph.D. degree in robotics, industrial automation systems, and bioengineering.

His main research interests are in the area of design and development of wearable electronical devices for biomechanical and multimedial application.

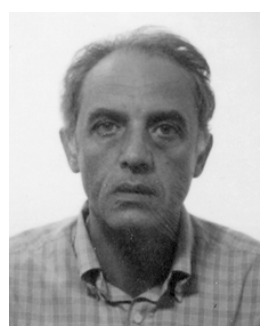

Danilo De Rossi received the degree in chemical engineering from the University of Genova, Genova, Italy, in 1976.

From 1976 to 1981, he was Researcher with the Institute of Clinical Physiology of C.N.R. He worked in France, USA, Brazil, and Japan. Since 1982, he has been with the School of Engineering, University of Pisa, Pisa, Italy, where is presently Full Professor of bioengineering. His scientific activities are related to the physics of organic and polymeric materials and to the design of sensors and actuators for bioengineering and robotics. He is the author of over 150 technical and scientific publications. 